process which predicts the status of individuals in year $i+1$ from their status (category) in year i. Historical data is used to estimate the transition probabilities which are modelled using a multinomial trend model. Confidence intervals are calculated using boot strap procedures.

Results By 2020 there will be a 54\% increase in the number of individuals who are receiving ART and a $42 \%$ increase in the number of individuals under CD4 monitoring. Results for individual HIV risk groups predict increases of at least 34\%, 77\% and $35 \%$ for heterosexuals, people who inject drugs and men who have sex with men, respectively.

Discussion/conclusion With such large increases in the number of people who are under CD4 monitoring and receiving ART, NHS boards will need to plan ahead to ensure they have adequate resources to treat those in need.

\section{P92 A COMPARISON OF BLOOD AND SALIVA SAMPLING FOR HOME HIV TESTING}

Lauren Bull*, Marco Rossi, Alan McOwan. Chelsea and Westminster Hospital, London, UK

\subsection{6/sextrans-2015-052126.135}

Background HIV home sampling offers an acceptable and convenient method for HIV testing and may provide a practical solution for increasing testing in high risk groups. However, we are unaware of any data comparing the effectiveness of different sampling methods. From August 2013 users of our online HIV testing service were offered an informed choice between blood and saliva HIV sampling.

Method We interrogated the database of all HIV home sampling requests and analysed any differences in demographics and return rates for both blood and saliva samples.

Results Between 15.8.13 and 31.11.14, 14312 home tests were requested. Blood tests were preferentially chosen $(9532,66.6 \%$ vs $4780,33.4 \%) .7257$ samples $(50.7 \%)$ were returned, this encompassed 4758 blood samples and 2499 saliva samples (49.9\% of requested blood samples vs $52.2 \%$ of requested saliva samples $\mathrm{p}=0.01)$. The service is predominantly aimed at men who have sex with men and of the returned samples the majority were from men $(6416,84.7 \%)$ Men were significantly statistically more likely to request blood samples than women $(67 \%$ vs $51 \%, \mathrm{p}<0.00001)$. In total there were 123 reactive samples (1.7\%, 116 men, 7 women), 82 from blood samples (77 men, 5 women) 41 from saliva (39 men, 2 women).

The average age of all requests was 30.3 years, 30.8 years in persons who returned samples and 29.7 years for those who did not $(p<0.00001)$. There was a significant difference in the ages of people requesting saliva versus blood samples (29.7 years vs 30.6 years $p<0.0001$ ). The average age of persons with negative samples was 30.8 years vs. 33.0 years in those with positive samples $(\mathrm{p}<0.05)$. The median number of days from when the sample was ordered to when it was collected back was 6 days in all groups (negative samples, reactive samples, men, women, blood and saliva).

Discussion Despite being more invasive when given an informed choice, more people chose blood over saliva sampling. However saliva samples were more likely to be returned. Women were statistically more likely than men to choose saliva sampling. There was no difference in the length of time it took to return reactive and negative samples.

\section{P93 HIV TESTING IN AN INTEGRATED SEXUAL HEALTH SERVICE}

Mamatha Oduru*, Matthew Hamill, Nisha Pal, Noreen Desmond. The Garden Clinic, Slough, UK

10.1136/sextrans-2015-052126.136

\begin{tabular}{|c|c|c|c|c|}
\hline Patient group & Codes & Week 1 & Week 2 & Week 1 and week 2 comparison \\
\hline \multirow[t]{4}{*}{ Total sample population $\mathrm{N}=205$} & & $\mathrm{~N}=114$ & $N=91$ & \\
\hline & $\mathrm{P} 1 \mathrm{~A}+\mathrm{T} 4$ & $61.4 \%$ & $65.9 \%$ & $4.5 \%$ increase \\
\hline & P1B & $25.4 \%$ & $23.1 \%$ & $2.3 \%$ decrease \\
\hline & P1C & $13.2 \%$ & $11 \%$ & $2.2 \%$ decrease \\
\hline \multirow[t]{4}{*}{ Total GU presentations N = $126(61 \%)$} & & $N=72$ & $N=54$ & \\
\hline & $\mathrm{P} 1 \mathrm{~A}+\mathrm{T} 4$ & $76.4 \%$ & $87 \%$ & $10.6 \%$ increase \\
\hline & P1B & $18.1 \%$ & $11.1 \%$ & $7 \%$ decrease \\
\hline & P1C & $5.6 \%$ & $1.9 \%$ & $3.7 \%$ decrease \\
\hline \multirow[t]{4}{*}{ Total contraception presentations $\mathrm{N}=67(33 \%)$} & & $N=33$ & $\mathrm{~N}=34$ & \\
\hline & $\mathrm{P} 1 \mathrm{~A}+\mathrm{T} 4$ & $30.3 \%$ & $29.4 \%$ & $0.9 \%$ decrease \\
\hline & P1B & $42.4 \%$ & $44 \%$ & $1.6 \%$ increase \\
\hline & P1C & $27.3 \%$ & $26.5 \%$ & $0.8 \%$ decrease \\
\hline \multirow[t]{4}{*}{ Total combined GU and contraception presentations N $=12(6 \%)$} & & $N=9$ & $N=3$ & \\
\hline & $\mathrm{P} 1 \mathrm{~A}+\mathrm{T} 4$ & $55.6 \%$ & $100 \%$ & $44.4 \%$ increase \\
\hline & P1B & $22.2 \%$ & $0 \%$ & N/A \\
\hline & P1C & $22.2 \%$ & $0 \%$ & N/A \\
\hline Comparative percentage accepting and declining HIV tests in GU v contraception & $\mathrm{P} 1 \mathrm{~A}+\mathrm{T} 4$ & & & GU $=81 \%$ \\
\hline \multirow[t]{3}{*}{ sub-groups } & (accept) & & & Contraception $=30 \%$ \\
\hline & P1B & & & $\mathrm{GU}=15.1 \%$ \\
\hline & (decline) & & & Contraception $=43.3 \%$ \\
\hline \multicolumn{5}{|l|}{ SHAAPT HIV codes: } \\
\hline \multicolumn{5}{|l|}{$\mathrm{T} 4+\mathrm{P} 1 \mathrm{~A}=$ HIV test done } \\
\hline \multicolumn{5}{|l|}{ P1B $=$ HIV test offered + declined } \\
\hline P1C $=$ HIV test inappropriate & & & & \\
\hline
\end{tabular}


Background/introduction National standards recommend eighty percent of new sexual health patients should have an HIV test. Thames Valley data from 2013 highlighted lower uptake of HIV testing in the region's only integrated sexual health service (SHS) compared to two local non-integrated services.

Aim(s)/objectives This audit measured differences in HIV testing uptake between genitourinary (GU) and contraception consultations in an integrated SHS and assessed the impact of a publicity campaign.

Methods SHHAPT codes and demographics were collected from all new patients over two weeks; non-coded patients were excluded. Retrospective case-note review differentiated GU from contraception presentations. 'National HIV testing week' posters were displayed in week 2. Data were analysed in Microsoft Excel.

Results Total sample size was 205 patients (week 1, $\mathrm{N}=114$, week $2, \mathrm{~N}=91$ ). $63 \%$ were female and $96 \%$ heterosexual. Age range was 14 to 83 (mean 31, standard deviation 13), with 36 countries of birth. Patients presented for GU issues $(\mathrm{N}=126$; $61 \%)$, contraception $(\mathrm{N}=67 ; 33 \%)$ and combined $(\mathrm{N}=12$; $6 \%)$. HIV uptake differed between GU and contraception groups (81\% v 30\%). Between weeks 1 and 2, testing uptake increased by $4.5 \%$ in the total population and $10.6 \%$ in the GU group with minimal change in the contraception group.

Discussion/conclusion HIV testing uptake is higher in GU presentations compared to contraception presentations. This large discrepancy impacts overall testing figures. A publicity campaign may have increased GU uptake but had no impact on contraception consultations. Targeted education and opt out testing should be considered in integrated services.

\section{P94 MORTALITY IN HIV POSITIVE PATIENTS IN A LARGE INNER CITY TEACHING HOSPITAL}

Eleanor Hamlyn*, Rebecca Simons, Ranjababu Kulasegaram. St Thomas' Hospital, London, UK

\subsection{6/sextrans-2015-052126.137}

Background/introduction With the advent of highly active antiretroviral therapy (HAART) mortality among HIV positive patients has fallen significantly. Mortality review is important to target care and interventions appropriately.

Methods We reviewed mortality data from 2013 to 2014 for patients under the care of the HIV team at an inner city teaching hospital. There were 39 deaths in our cohort of 3400 patients.

Results Our cohort matched demographic data for people living with HIV in the UK in most respects: male to female ratio was approximately 7:3, 56\% were Caucasian, 33\% Black African. $21 \%$ of patients had acquired HIV via intravenous drug use (although only $2 \%$ of people living with HIV nationally are drug users). $28 \%$ were men who have sex with men. The median age of death was 47 . The most common cause of death was malignancy (44\%) followed by sepsis and ischaemic heart disease. Those with a CD4 count $<200$ at diagnosis survived on average 5.7 years before death. Those with a CD4 count $>200$ at diagnosis survived 9.7 years on average.

Discussion/conclusion In the post-HAART era, the majority of deaths in people with HIV are not HIV related. Nine patients, however, had an AIDS defining malignancy and three had active opportunistic infections. In the era of HAART, screening for chronic disease and malignancy is vital. Our data suggest that intravenous drug use is a significant factor in people dying at a younger age with HIV. There remains a correlation between late diagnosis and increased risk of death.

\section{\begin{tabular}{|l|l}
\hline P95 THE ABILITY OF THE ALERETM HIVCOMBO POINT-OF \\
\hline
\end{tabular} CARE TEST TO DETECT ACUTE HIV INFECTION}

Naomi Fitzgerald*, Maria Cross, Siobhan O'Shea, Julie Fox. Guy's and St Thomas' Hospital, London, UK

\subsection{6/sextrans-2015-052126.138}

Background/introduction Detection of acute HIV infection is important in preventing HIV transmission and for consideration of early antiretroviral therapy. Fourth generation (4G) HIV tests detect p24 antigen and HIV antibody and should detect acute HIV infection prior to the development of antibodies. An early version fourth generation (4G) point-of-care (POCT) test demonstrated low levels of sensitivity for p24Ag.

Aim(s)/objectives To assess the ability of the new Alere ${ }^{\mathrm{TM}}$ HIV Combo 4G POCT to detect p24 antigen in patients with laboratory confirmed p24 antigenaemia.

Methods P24 antigen positive serum samples were tested using the Alere ${ }^{\mathrm{TM}}$ HIV-Combo POCT and read at 20 and $40 \mathrm{~min}$. One sample gave an invalid result and was excluded. P24 antigen levels from the VIDAS quantitative HIV p24 11 assay, used as routine HIV confirmatory tests by our laboratory, were recorded for comparison.

Results Twenty-four out of 27 samples (89\%) were p24 antigen positive at $20 \mathrm{~min}$ and $25 / 27(93 \%)$ samples were positive at $40 \mathrm{~min}$. There were two false negative samples, shown to have the lowest levels of p24 antigen $(27.6$ and $8.3 \mathrm{pg} / \mathrm{ml})$ of the 27 samples. The mean p24 antigen level with the VIDAS quantitative HIV p24 11 assay for the cohort was 236.2 (Range 8.3$>400 \mathrm{pg} / \mathrm{ml}$ ). The Alere ${ }^{\mathrm{rm}}$ HIV Combo POCT detected all P24 antigen at levels $>30 \mathrm{pg} / \mathrm{ml}$.

Discussion/conclusion The Alere ${ }^{\mathrm{rM}}$ HIV Combo POCT has $89 \%$ sensitivity for p24 antigen at $20 \mathrm{~min}$ and $93 \%$ at $40 \mathrm{~min}$. These preliminary results suggest that the new Alere ${ }^{\mathrm{TM}}$ HIV Combo POCT may be able to detect early infection adequately.

\section{P96 ACCESS OF LEVEL 2 SEXUAL HEALTH SERVICES BY MEN WHO HAVE SEX WITH MEN: WHO GOES AND WHAT SERVICES DO THEY GET?}

Helen Mebrahtu*, Bersabeh Sile, Hamish Mohammed, John Were, Mandy Yung, Gwenda Hughes. Public Health England, London, UK

\subsection{6/sextrans-2015-052126.139}

Background Men who have sex with men (MSM) bear a disproportionate burden of sexually transmitted infections (STIs) including HIV. While routine STI surveillance data indicate MSM regularly access genitourinary medicine (GUM) services for their sexual health care, the extent to which MSM attend non-specialist Level 2 sexual health services is unclear. We investigated access of Level 2 services by MSM in England.

Methods We used provisional data from the GUM Clinic Activity Dataset (GUMCADv2) to compare the characteristics, service usage and outcomes between MSM accessing GUM and Level 2 services who reported data in 2013.

Results Of all male attendances where sexual orientation was recorded, $12.3 \%(6,957 / 57,048)$ of Level 2 attendances were among MSM compared to $26.3 \%(299,456 / 1,139,424)$ of GUM attendances $(\mathrm{p}<0.001)$. MSM attending Level 2 compared to 